\title{
Primary adrenal sarcomatoid carcinoma metastatic to the lung: Case report and review of the literature
}

\author{
CHUANGZHI ZHU ${ }^{1-3}$, AIPING ZHENG ${ }^{4}$, XIANGMING MAO ${ }^{1,2}$, BENTAO SHI $^{1,2}$ and XIANXIN LI ${ }^{1,2}$ \\ ${ }^{1}$ Department of Urology, Peking University Shenzhen Hospital; \\ ${ }^{2}$ The Guangdong and Shenzhen Key Laboratory of Male Reproductive Medical and Genetics, Institute of Urology, \\ Shenzhen PKU-HKUST Medical Center, Peking University Shenzhen Hospital, Shenzhen, Guangdong 518036; \\ ${ }^{3}$ Shantou University Medical College, Shantou, Guangdong 515041; ${ }^{4}$ Department of Pathology, \\ Peking University Shenzhen Hospital, Shenzhen, Guangdong 518036, P.R. China
}

Received January 12, 2015; Accepted February 11, 2016

DOI: $10.3892 / \mathrm{ol} .2016 .4342$

\begin{abstract}
Adrenal sarcomatoid carcinoma is a rare adrenal carcinoma. To the best of our knowledge, only 11 cases have been reported since 1987. Adrenal sarcomatoid carcinoma presents a diagnostic challenge due to its atypical symptoms and histological patterns. At the time of diagnosis, a large percentage of patients are already at the metastatic stage and succumb within a few months. The present study reports a case of a 59-year-old man presenting with asthenia and weight loss with adrenal sarcomatoid carcinoma metastatic to the lung. A computed tomography (CT) scan and ultrasonography of the patient's abdomen suggested a large homogeneous mass in the right adrenal gland, and a CT scan of his chest suggested lung metastasis. Right adrenalectomy was performed. Histological examination revealed that the tumor was composed of sarcomatous and carcinomatous differentiation elements. Immunohistochemical examination revealed tumor cell positivity for vimentin and cytokeratin. At the 6-month follow-up the patient exhibited no disease progression and refused further proposed treatment. The patient was alive at the time of writing the current report. The present case report additionally reviews the literature, for the purpose of raising awareness of these rare lesions and assisting in achieving accurate diagnoses and effective treatment.
\end{abstract}

\section{Introduction}

Adrenocortical carcinoma is a rare malignant tumor, accounting for $\sim 0.02 \%$ of global malignancies (1). Adrenocortical

Correspondence to: Dr Bentao Shi or Professor Xianxin Li, Department of Urology, Peking University Shenzhen Hospital, 1120 Lianhua Road, Shenzhen, Guangdong 518036, P.R. China E-mail: shibentaopku@126.com

E-mail: xianxinli@163.com

Key words: adrenal, sarcomatoid carcinoma, adrenocortical carcinoma carcinoma has a bimodal distribution by age, with one peak age before 5 years, and the other between 40 and 50 years old, as well as a female predominance (2). Sarcomatoid carcinomas, which are comprised of sarcomatous and carcinomatous differentiation components, are a notable kind of malignant tumor, commonly occurring in the digestive (3) and respiratory (4) tracts, and breasts (5). This tumor is rarely observed in the adrenal glands. Primary adrenal sarcomatoid carcinoma, an extremely uncommon, aggressive malignant tumor, constitutes a subgroup of adrenocortical carcinomas $(6,7)$. The typical symptoms associated with the disease are abdominal pain, weight loss and lumbago, and surgery is the major modality for the treatment of primary adrenal sarcomatoid carcinoma. Primary adrenal sarcomatoid carcinoma is likely to exhibit distant metastasis and patients often succumb to disease within 2 years following surgery (8). The first report of primary adrenal sarcomatoid carcinoma was in 1989 by Collina et al (6). At the time of writing the present study, to the best of our knowledge only 11 cases had previously been reported (6,9-18). The majority of those patients succumbed to disease within a year, due to local recurrence or de novo metastases. Timely and accurate diagnosis and effective treatments are therefore required. The present study reports a case of primary sarcomatoid carcinoma with lung metastasis, and reviews the previous literature in order to identify the optimal management practices.

\section{Case report}

A 59-year-old man presented to the Department of Urology, Peking University Shenzhen Hospital (Shenzhen, China) for examination on April 28, 2014, with a 4-month history of asthenia and weight loss, without fever, abdominal pain or other notable medical history. The patient provided written informed consent for the publication of their data. Upon initial physical examination, the patient's blood pressure was $115 / 75 \mathrm{mmHg}$, however, he exhibited no other symptoms that could be due to excessive levels of steroidal hormones or catecholamine. Abdominal palpation revealed no masses. Aldosterone (56.38 pg/ml; normal, 23.50-106.60 pg/ml) and cortisol (8 AM, $13.96 \mu \mathrm{g} / \mathrm{dl}$; normal, 7.20-18.20 $\mu \mathrm{g} / \mathrm{dl}$; 4 AM, 

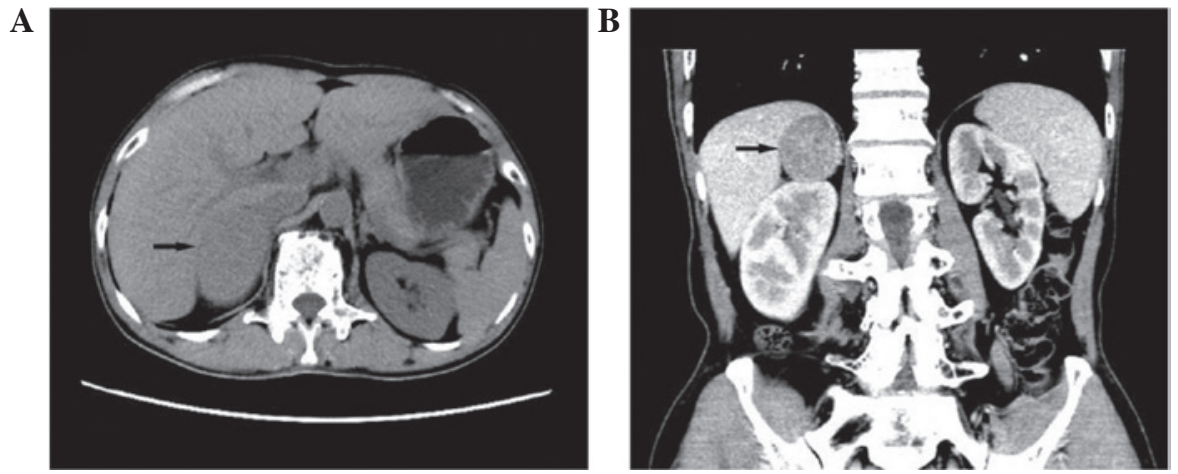

Figure 1. Abdominal computed tomography scan. (A) Axial and (B) coronal views revealed a large, homogeneously dense, space-occupying mass located in the region of the right adrenal gland was adjacent to the liver, inferior vena cava and right renal vein.
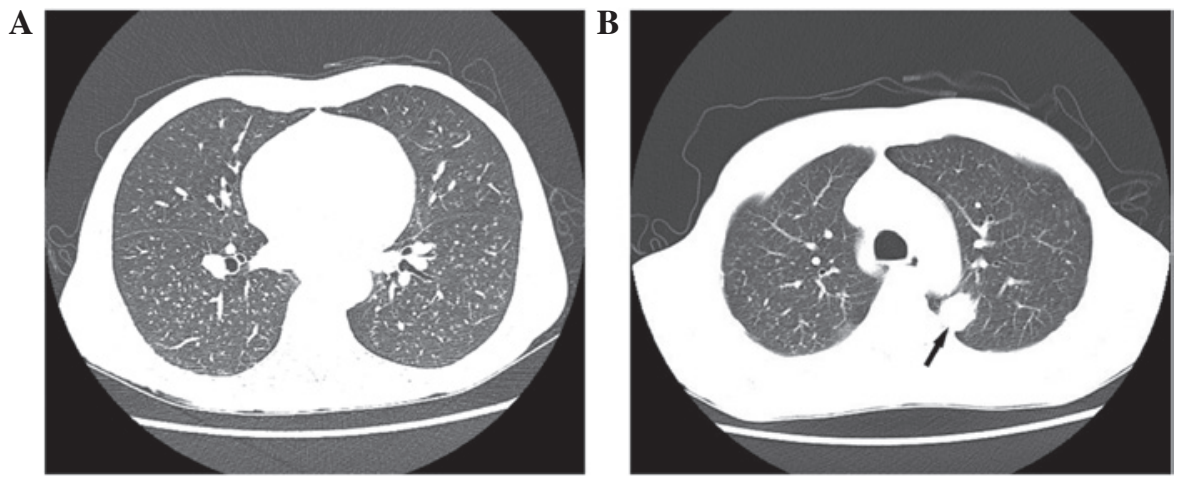

Figure 2. Chest computed tomography scan showing (A) small nodes with diffused distribution in the lungs and (B) a large, well-defined mass in the left lung.

$6.61 \mu \mathrm{g} / \mathrm{dl}$; normal, 2.75-6.65 $\mu \mathrm{g} / \mathrm{dl} ; 12 \mathrm{AM}, 0.96 \mu \mathrm{g} / \mathrm{dl}$; normal, $0.00-1.54 \mu \mathrm{g} / \mathrm{dl})$ levels were within the normal limits. Initial blood tests revealed low hemoglobin levels $(71 \mathrm{~g} / \mathrm{l}$; normal, 120-160 g/l). The serum levels of carbohydrate antigen 125 exceeded the normal limits $[211.10$ units $(\mathrm{U}) / \mathrm{ml}$; normal, $<35.00 \mathrm{U} / \mathrm{ml}$, while those of carbohydrate antigen 19-9 (2.05 U/ml; normal, $<37.00 \mathrm{U} / \mathrm{ml})$, carbohydrate antigen $15-3$ (8.80 U/ml; normal, <31.30 U/ml), $\alpha$-fetoprotein $(1.15 \mathrm{ng} / \mathrm{ml}$; normal, $<13.40 \mathrm{ng} / \mathrm{ml})$, carcinoembryonic antigen $(0.74 \mathrm{ng} / \mathrm{ml}$; normal, $0.00-5.00 \mathrm{ng} / \mathrm{ml}$ ) and squamous cell carcinoma antigen $(0.7 \mathrm{ng} / \mathrm{ml}$; normal, $0.0-1.5 \mathrm{ng} / \mathrm{ml})$ were within the normal ranges. Abdominal ultrasonography (LOGIQ E9; GE Healthcare, Little Chalfont, UK) revealed a right, capsuled adrenal mass of $6.5 \times 3.7 \mathrm{~cm}$, with no obvious calcification or liquefaction. A computed tomography (CT) scan, using a Sensation 16 multiple detector CT (Siemens Healthcare, Erlangen, Germany), of the abdomen indicated a well-defined, homogeneously dense, $6 \times 4 \mathrm{~cm}$ tumor in the right adrenal gland (Fig. 1). The tumor was adjacent to the liver, inferior vena cava and right renal vein (Fig. 1). An additional chest CT suggested bilateral lung metastases (Fig. 2). The preoperative diagnosis was adrenal malignancy. The patient underwent right adrenalectomy. The surgery revealed an entirely capsuled tumor adhering to the liver.

Macroscopic examination revealed the tumor to be soft and $\sim 5 \mathrm{~cm}$ in diameter, with its dissected surface revealing a white-to-grey rim and pink soft node within a well-defined capsule. Following resection, tissues were sent to the Department of Pathology, Peking University Shenzhen Hospital for pathological and immunohistochemical analysis. Formalin (Guangzhou Kaixiu Co., Ltd, Guangzhou, China)-fixed, paraffin (Beijing Solarbio Science \& Technology Co., Ltd., Beijing, China)-embedded resected tissues ( $4 \mu \mathrm{m}$ pathological sections) were used for histological staining with hematoxylin and eosin (Beijing Solarbio Science \& Technology Co., Ltd.). Tissues were visualized using a microscope (DMIL-LED; Leica Microsystems, Wetzlar, Germany). Microscopic examination revealed extensive growth with epithelial and spindle cell components (Fig. 3). Tumor cells were observed to be large and stratified in sheets. Hyperchromatic nuclei and atypical mitoses were present. Formalin-fixed, paraffin-embedded tissues ( $4 \mu \mathrm{m}$ pathological sections) were used for immunohistochemical analysis with rabbit anti-vimentin monoclonal (catalog no., RMA-0547; 1:500; Fuzhou Maixin Biotech. Co., Ltd., Fuzhou, China) and mouse anti-cytokeratin monoclonal (pan-Cytokeratin Immunohistochemistry Detection kit; catalog no., IPM067; 1:500; Guangzhou An Biping (LBP) Pharmaceutical Technology Co., Ltd., Guangzhou, China) primary antibodies. Immunohistochemistry revealed tumor cells that were diffused and strongly positive for vimentin and cytokeratin (Fig. 3). Immunohistochemical stains for smooth muscle actin, S-100 protein, $\alpha$-inhibin, human melanoma black 45, melan-A protein, synaptophysin, chromogranin A, thyroid transcription factor-1, desmin and myogenin were negative for carcinomatous and sarcomatous components (Fig. 3). The final pathological diagnosis was primary sarcomatoid carcinoma of the right adrenal gland. Postoperatively, the patient refused adjuvant chemotherapy and was discharged from hospital. 


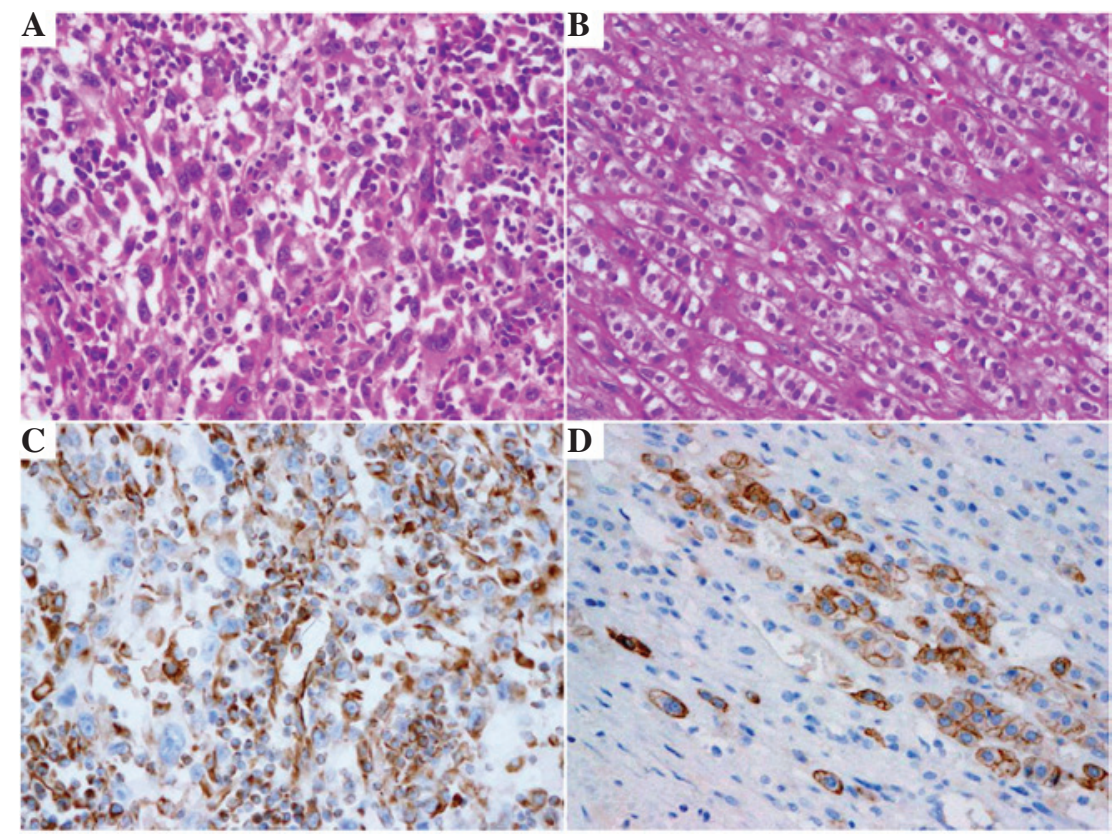

Figure 3. Histopathological examination of the adrenal sarcomatoid carcinoma. Hematoxylin and eosin stain revealing (A) sarcomatous component composed of uniform spindle cells and (B) carcinomatous component composed of epithelial cells. The cytoplasm of (C) the sarcomatous cells stained positive for vimentin, while that of (D) the carcinomatous cells stained positive for cytokeratin. Magnification, x200.

At the time of writing, 6 months postoperatively, the patient remained alive and, to the best of our knowledge, was not undergoing any further treatment or follow-up visits.

\section{Discussion}

Adrenocortical carcinoma is an uncommon, aggressive tumor, occurring annually at the rate of 0.7-2.0/1,000,000 of the global population (19), and is known to metastasize through the lymphatic and circulatory systems, as well as through implantation (20). It has been reported that $21-39 \%$ of all patients have distant metastasis at the time of diagnosis, which emphasizes the urgent need for novel treatment methods $(2,21)$. Adrenocortical carcinoma typically metastasizes to the lung, liver, peritoneum and pleura, lymph nodes, and bones (22). Prognosis is considerably poor, and the 5-year overall survival rate of advanced adrenocortical carcinoma is $<30 \%$ (23). Even with favorably performed radical resections, the majority of patients succumb to local recurrence or de novo metastases $(2,8,21)$.

Adrenal sarcomatoid carcinoma is a rare adrenocortical carcinoma subgroup (7). The 2004 WHO classification defines it as a 'sarcomatoid carcinoma' (7). It rarely occurs in the urinary system and morbidity has been observed to be $<3 \%$ for patients with urological malignancy (24). Sarcomatoid carcinomas are described as malignant tumors comprised of epithelial and mesenchymal components, which may malignantly differentiate into cartilage, fibrosarcoma, bone or skeletal muscle (25-27). Adrenal sarcomatoid carcinomas are more likely to occur in the kidney or bladder, rather than the adrenal glands (17). These carcinomas may be classified as either functional or nonfunctional tumors (1). Patients with functional tumors may present with symptoms caused by excessive hormones, including the following: i) Cushing's syndrome; ii) severe hypertension; iii) male feminization or iv) female masculinization. Patients with nonfunctional tumors may present with symptoms including the following: i) Weight loss; ii) abdominal masses; iii) abdominal pain; iv) low fever and v) asthenia (1).

To the best of our knowledge, the present case report describes the 12th reported case of primary adrenal sarcomatoid carcinoma and reviews the previous literature (Table I) (6,9-18). Data collected from the present patient, as well as from the previous 11 reported cases identified in the literature, revealed that the patient age range at the time of presentation with adrenal sarcomatoid carcinoma was 29-79 years, with a mean age of 55.75 years $(6,9-18)$. The female:male ratio in this small sample size was $1: 1$. The majority of studies have demonstrated that women are more susceptible to the disease $(1,28)$. Based on the collected data, there appear to be no significant differences in morbidity rates between male and female patients (Table 1). In addition, based on the collection data, middle-aged and elderly patients should be considered high risk.

Upon initial presentation with primary adrenal sarcomatoid carcinoma, patients typically complain of abdominal pain or discomfort, lumbago and weight loss (6,9-18). Only $2 / 12$ reported cases were functional tumors $(11,12)$. The hormone levels of the present patient were within the normal limits. It is likely that, at the time of diagnosis, the patients in all 12 cases were at an advanced disease stage and had distant metastases. At diagnosis, 2 of the reported cases presented with inferior vena cava invasion and liver metastasis $(12,13)$, while the present patient presented with lung metastasis preoperatively. All 12 cases were surgically treated. Only 3 of these cases received combined adjuvant chemotherapy, including the present study $(6,14)$. The majority of patients succumbed to recurrence or metastasis complications within a year of surgery. The patient in the present study, along with 7 others from the literature $(6,9,13,14,16-18)$, demonstrated a 


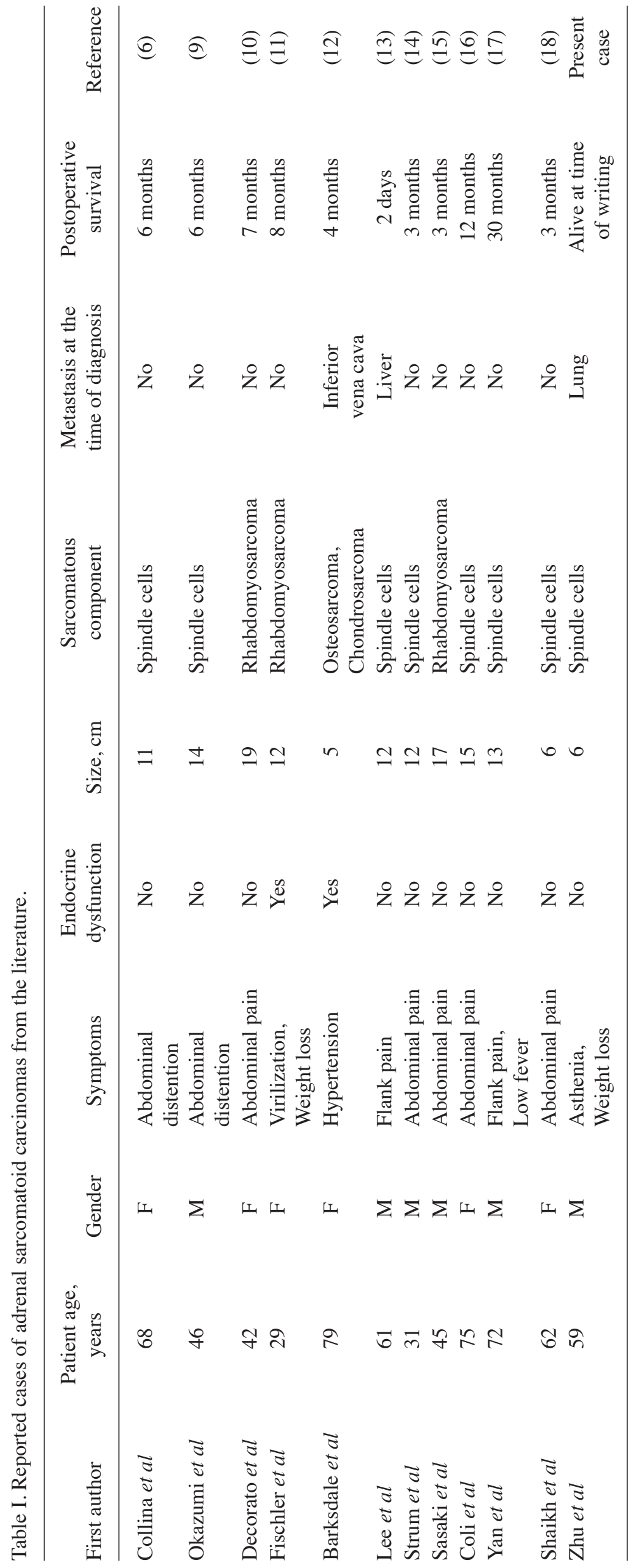


sarcomatous component containing spindle cells, while 4 had rhabdomyosarcoma $(10-12,15)$, or a mixture of osteosarcoma and chondrosarcoma.

Adrenal sarcomatoid carcinomas tend to be aggressive, and patients often present with extensive locoregional spread at diagnosis (1). Preoperative diagnosis is challenging, which reduces treatment effectiveness and directly influences prognosis. A previous study demonstrated that endocrine assays and CT scans are effective modalities for diagnosis and tumor location confirmation (29). Dynamic enhanced multi-detector row CT scans may reveal tumor composition and blood supply (29). Histopathology and immunohistochemistry must be performed to confirm a diagnosis (30). Surgical excision, including complete metastatic resection, is currently the most effective treatment, while postoperative adjuvant chemotherapy may decrease relapse and metastasis rates $(31)$. Previous case reports $(6,14)$ and the present study appear to suggest that chemotherapy has no significant effect on adrenal sarcomatoid carcinomas. Additional research is required in order to confirm the efficacy of chemotherapy. Mitotane is recommended as the preferred treatment for patients with advanced adrenocortical carcinoma (32). It works primarily by inducing adrenal cortex degeneration and necrosis. Chemotherapy combined with mitotane has been demonstrated to be more effective than chemotherapy alone (33). In these treatment regimes, mitotane serum levels must be monitored. Molecularly-targeted tumor therapy has previously been studied with findings indicating that certain oncogenes, growth factors and tumor-suppressor genes are associated with adrenocortical carcinoma tumorigenesis. It should be noted that epidermal growth factor receptor (EGFR) is overexpressed in the majority of adrenocortical carcinomas (34), and has been demonstrated to have a significant role in sarcomatoid carcinomas $(35,36)$. Anti-EGFR therapy may comprise a feasible approach for the improvement of prognosis in patients with adrenal sarcomatoid carcinomas.

Adrenal sarcomatoid carcinomas are rare but aggressive tumors, and are associated with poor prognosis and a lack of effective treatment options. At present, only postoperative immunohistochemical examination is able to provide a definitive diagnosis. Surgery is the single, yet ineffective, treatment option for tumors at present. Adjuvant chemotherapy is ineffective for advanced adrenal sarcomatoid carcinoma; therefore, additional research is required in order to explore targeted therapy for the treatment of this rare, but fatal, type of tumor.

\section{Acknowledgements}

The present study was supported by the Medical Scientific Research Foundation of Guangdong Province, China (grant. no. A2014653) and the Science and Technology project in Shenzhen, Guangdong Province, China (grant. no. 201302052).

\section{References}

1. Roman S: Adrenocortical carcinoma. Curr Opin Oncol 18 36-42, 2006.
2. Ng L and Libertino JM: Adrenocortical carcinoma: Diagnosis, evaluation and treatment. J Urol 169: 5-11, 2003.

3. Weidner N: Sarcomatoid carcinoma of the upper aerodigestive tract. Semin Diagn Pathol 4: 157-168, 1987.

4. Zarbo RJ, Crissman JD, Venkat H and Weiss MA: Spindle-cell carcinoma of the upper aerodigestive tract mucosa. An immunohistologic and ultrastructural study of 18 biphasic tumors and comparison with seven monophasic spindle-cell tumors. Am J Surg Pathol 10: 741-753, 1986.

5. Oberman HA: Metaplastic carcinoma of the breast. A clinicopathologic study of 29 patients. Am J Surg Pathol 11: 918-929, 1987.

6. Collina G, Maldarizzi F, Betts CM and Eusebi V: Primary sarcomatoid carcinoma of the adrenal gland. First case report. Virchows Arch A Pathol Anat Histopathol 415: 161-167, 1989.

7. DeLellis RA, Lloyd RV, Heitz PU and Eng C (eds): World Health Organization Classification of Tumors. Pathology and Genetics of Tumours of Endocrine Organs. Vol. 8. IARC Press, Lyon, France, 2004

8. Tauchmanovà L, Colao A, Marzano LA, Sparano L, Camera L, Rossi A, Palmieri G, Marzano E, Salvatore M, Pettinato G, et al: Andrenocortical carcinomas: Twelve-year prospective experience. World J Surg 28: 896-903, 2004.

9. Okazumi S, Asano T, Ryu M, Nagashima T, Odaka M, Isono K and Nishizawa T: Surgical resection of adrenal carcinoma extending into the vena cava, right atrium and ventricle: Case report and review of the literature. Nihon Geka Gakkai Zasshi 88: 231-238, 1987 (In Japanese).

10. Decorato JW, Gruber H, Petti M and Levowitz BS: Adrenal carcinosarcoma. J Surg Oncol 45: 134-136, 1990.

11. Fischler DF, Nunez C, Levin HS, McMahon JT, Sheeler LR and Adelstein DJ: Adrenal carcinosarcoma presenting in a woman with clinical signs of virilization. A case report with immunohistochemical and ultrastructural findings. Am J Surg Pathol 16: 626-631, 1992.

12. Barksdale SK, Marincola FM and Jaffe G: Carcinosarcoma of the adrenal cortex presenting with mineralocorticoid excess. Am J Surg Pathol 17: 941-945, 1993.

13. Lee MS, Park IA, Chi JG, Ham EK, Lee KC and Lee CW: Adrenal carcinosarcoma-a case report. J Korean Med Sci 12: 374-377, 1997.

14. Sturm N, Moulai N, Laverrière MH, Chabre O, Descotes JL and Brambilla E: Primary adrenocortical sarcomatoid carcinoma: Case report and review of literature. Virchows Arch 452: 215-219, 2008.

15. Sasaki K, Desimone M, Rao HR, Huang GJ and Seethala RR: Adrenocortical carcinosarcoma: A case report and review of the literature. Diagn Pathol 5: 51, 2010.

16. Coli A, Di Giorgio A, Castri F, Destito C, Marin AW and Bigotti G: Sarcomatoid carcinoma of the adrenal gland: A case report and review of literature. Pathol Res Pract 206: 59-65, 2010.

17. Yan JJ, Sun AJ, Ren Y and Hou C: Primary adrenocortical sarcomatoid carcinoma: Report of a case. Can Urol Assoc J 6: E189-E191, 2012

18. Shaikh AS, Bakhshi GD, Khan AS, Jamadar NM, Nirmala AK and Raza AA: Primary adrenal sarcomatoid carcinoma. Clin Pract 4: 604, 2014.

19. Fassnacht M, Libé R, Kroiss M and Allolio B: Adrenocortical carcinoma: A clinician's update. Nat Rev Endocrinol 7: 323-335, 2011.

20. Jin Z, Ogata S, Tamura G, Katayama Y, Fukase M, Yajima M and Motoyama T: Carcinosarcomas (malignant mullerian mixed tumors) of the uterus and ovary: A genetic study with special reference to histogenesis. Int J Gynecol Pathol 22: 368-373, 2003.

21. Icard P, Goudet P, Charpenay C, Andreassian B, Carnaille B, Chapuis Y, Cougard P, Henry JF and Proye C: Adrenocortical carcinomas: Surgical trends and results of a 253-patient series from the French Association of Endocrine Surgeons study group. World J Surg 25: 891-897, 2001.

22. Hutter AM Jr and Kayhoe DE: Adrenal cortical carcinoma. Clinical features of 138 patients. Am J Med 41: 572-580, 1966.

23. Bourdeau I, MacKenzie-Feder J and Lacroix A: Recent advances in adrenocortical carcinoma in adults. Curr Opin Endocrinol Diabetes Obes 20: 192-197, 2013.

24. Fleming S: Carcinosarcoma (mixed mesodermal tumor) of the ureter. J Urol 138: 1234-1235, 1987.

25. Beasley MB, Brambilla E and Travis WD: The 2004 World Health Organization classification of lung tumors. Semin Roentgenol 40: 90-97, 2005.

26. Craig JR, Peters RL and Edmondson HA: Tumors of the Liver and Intrahepatic Bile Ducts. Armed Forces Institute of Pathology, Washington, DC, pp185-186, 1989. 
27. Koss MN, Hochholzer L and Frommelt RA: Carcinosarcomas of the lung: A clinicopathologic study of 66 patients. Am J Surg Pathol 23: 1514-1526, 1999.

28. Dackiw AP, Lee JE, Gagel RF and Evans DB: Adrenal cortical carcinoma. World J Surg 25: 914-926, 2001.

29. Feng YC, Yang ZG, Chen TW, Su XY, Deng W and Wang QL: Adrenal sarcomatoid carcinoma: A rare case depicted on multi-detector row computed tomography. Indian J Med Sci 64: 37-40, 2010.

30. van't Sant HP, Bouvy ND, Kazemier G, Bonjer HJ, Hop WCJ, Feelders RA, de Herder WW and de Krijger RR: The prognostic value of two different histopathological scoring systems for adrenocortical carcinomas. Histopathology 51: 239-245, 2007.

31. Kendrick ML, Lloyd R, Erickson L, Farley DR, Grant CS, Thompson GB, Rowland C, Young WF Jr and van Heerden JA Adrenocortical carcinoma: Surgical progress or status quo? Arch Surg 136: 543-549, 2001.

32. Schteingart DE, Doherty GM, Gauger PG, Giordano TJ, Hammer GD, Korobkin M and Worden FP: Management of patients with adrenal cancer: Recommendations of an international consensus conference. Endocr Relat Cancer 12: 667-680, 2005.
33. Terzolo M, Daffara F, Ardito A, Zaggia B, Basile V, Ferrari L and Berruti A: Management of adrenal cancer: A 2013 update. J Endocrinol Invest 37: 207-217, 2014

34. Kamio T, Shigematsu K, Sou H, Kawai K and Tsuchiyama H: Immunohistochemical expression of epidermal growth factor receptors in human adrenocortical carcinoma. Hum Pathol 21: 277-282, 1990.

35. Wang X, MacLennan GT, Zhang S, Montironi R, Lopez-Beltran A, Tan PH, Foster S, Baldridge LA and Cheng L: Sarcomatoid carcinoma of the upper urinary tract: Clinical outcome and molecular characterization. Hum Pathol 40: 211-217, 2009.

36. Italiano A, Cortot AB, Ilie M, Martel-Planche G, Fabas T, Pop D, Mouroux J, Hofman V, Hofman P and Pedeutour F: EGFR and KRAS status of primary sarcomatoid carcinomas of the lung: Implications for anti-EGFR treatment of a rare lung malignancy. Int J Cancer 125: 2479-2482, 2009. 